\title{
Eye Movement Desensitisation and Reprocessing Treatment of Posttraumatic Stress Disorder, Comorbid Disorders and Personality Traits: A Case Series with 12 Month Follow Up
}

Laugharne $\mathrm{J}^{*}$, Kullack $\mathrm{C}$ and Stanley $\mathbf{S}$

Community, Culture and Mental Health Unit, School of Psychiatry and Clinical Neurosciences, University of Western Australia, Australia

\begin{abstract}
Eye movement desensitisation and reprocessing is an established treatment for posttraumatic stress disorder. Patients with posttraumatic stress disorder have elevated rates of comorbid psychiatric disorders and personality disorder. This paper reports on a series of seven cases of posttraumatic stress disorder, drawn from a secondary level care outpatient clinic, for which systematic clinical data was recorded using validated instruments, before and after treatment with eye movement desensitisation and reprocessing and at 12 month follow-up. All patients improved significantly in terms of posttraumatic stress disorder symptoms. There were significant reductions in paranoid, depressive and borderline traits post treatment and at follow-up. There was a reduction in comorbid psychiatric diagnoses and in personality disorder diagnoses post treatment and maintained at follow-up. These results suggest that eye movement desensitisation and reprocessing may be effective in treating comorbid disorders as well as PTSD and indicate a need for further studies with appropriate controls.
\end{abstract}

Keywords: Eye movement desensitization and reprogramming; Posttraumatic Stress Disorder; Personality Traits; Comorbid psychiatric disorders

\section{Introduction}

Eye movement desensitisation and reprocessing (EMDR) is an established treatment for post traumatic stress disorder (PTSD) [13]. It is well understood that individuals with PTSD have high rates of psychiatric comorbidities, particularly depressive disorders, anxiety disorders, substance misuse disorders and personality disorders [4,5]. There is, however, limited evidence outlining the effects of EMDR on comorbid psychiatric disorders or on personality traits and disorders when patients receive treatment for their PTSD symptoms. We have collected data pertinent to these issues on all patients treated primarily with EMDR at our Posttraumatic Stress Clinic at Fremantle Hospital, Western Australia since 2009. This clinic is a small research clinic staffed by a part time Psychiatrist and a part time Nurse Therapist. The clinic, although privately funded, is located at a major government and teaching hospital in an urban setting. Patients are referred to the clinic through local general practitioners, mental health teams, and medical teams. In this paper we report on a series of seven cases for whom systematic clinical data is available up to 12 months post-treatment with EMDR.

\section{Method}

Patients were assessed via clinical interview, of which all were supported by the use of validated instruments including the Mini International Neuropsychiatric Interview PLUS (MINI PLUS) [6], the PTSD checklist (civilian version) [7], the Hamilton Anxiety Scale (HAM-A) [8], the Hamilton Depression Scale (HAM-D) [9] the Dissociative Experiences Scale [10] and the Structured Clinical Interview for DSM IV Axis II Personality Disorders $2^{\text {nd }}$ version (SCID II) [11].

The MINI PLUS, a structured diagnostic interview, was developed jointly by clinicians and psychiatrists, and designed to assess for and diagnose 23 psychiatric disorders [6]. The MINI plus is a highly sensitive and specific screening tool adaptable to many clinical and research settings. Overall, the reliability and validity data of the MINI is very positive $[12,13]$.
The PTSD checklist is a brief self-report instrument used to assess the re-experiencing, avoidance and hyper-arousal symptoms of PTSD [7]. It is widely used in the clinical setting for tracking symptoms and as a diagnostic tool in research studies. As the PTSD Checklist (civilian version) does not allow for investigators to confirm that the traumatic event met the Criteria A component of the DSM IV PTSD diagnosis it is more useful when followed by a second tier diagnostic test [14] (such as the MINI Plus).

The HAM-A is a 14 items test used to measure the severity of overall anxiety, psychic anxiety, and somatic anxiety. It can be used in both adults and children populations, and as an outcome measure when assessing the impact of therapies and treatments [8]. Maier et al. [15] found that although the application of the HAM-A is limited in anxiolytic treatment studies, overall the reliability and validity of the HAM-A has proved to be sufficient.

The HAM-D is a 17 item questionnaire used to provide an indication of depression and as a guide to evaluate recovery [9]. Developed in the 1960's by Max Hamilton it has, of late, been subject to criticism and identified as being psychometrically and conceptually flawed [16]. Despite this, the HAM-D internal reliability is believed to be sufficient and is reported as one of the most commonly used depressive scales [17].

The DES was developed by Bernstein and Putnam [10] as a self-

*Corresponding author: Jonathan Laugharne, Community, Culture and Mental Health Unit, School of Psychiatry and Clinical Neurosciences, University of Western Australia, W block, L6, 1 Alma St Fremantle, WA 6160, Australia, Tel: +61 89431 3467; Fax: +61 89431 3407; E-mail: jonathan.laugharne@uwa.edu.au

Received September 19, 2013; Accepted October 28, 2013; Published October 30, 2013

Citation: Laugharne J, Kullack C, Stanley S (2013) Eye Movement Desensitisation and Reprocessing Treatment of Posttraumatic Stress Disorder, Comorbid Disorders and Personality Traits: A Case Series with 12 Month Follow Up. J Trauma Treat S4: 003. doi:10.4172/2167-1222.S4-003

Copyright: ( ) 2013Laugharne $\mathrm{J}$, et al. This is an open-access article distributed under the terms of the Creative Commons Attribution License, which permits unrestricted use, distribution, and reproduction in any medium, provided the original author and source are credited. 
Citation: Laugharne J, Kullack C, Stanley S (2013) Eye Movement Desensitisation and Reprocessing Treatment of Posttraumatic Stress Disorder, Comorbid Disorders and Personality Traits: A Case Series with 12 Month Follow Up. J Trauma Treat S4: 003. doi:10.4172/2167-1222.S4003

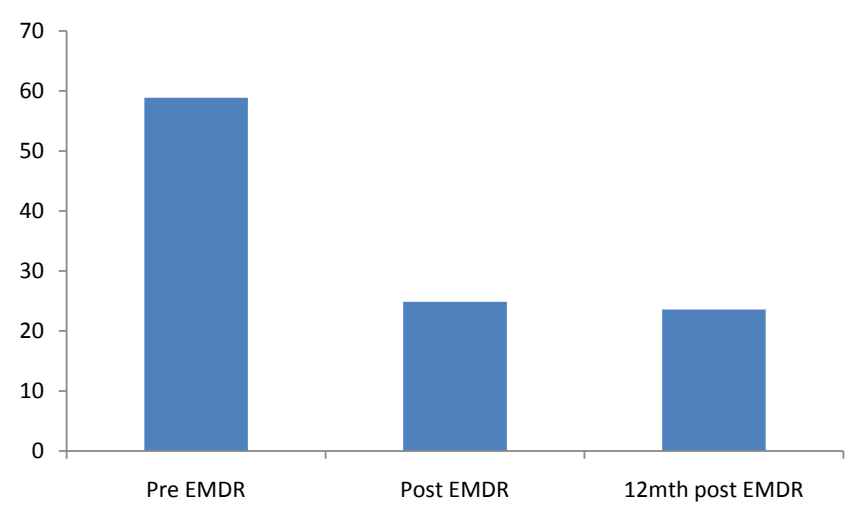

$\mathrm{EMDR}=$ Eye Movement Desensitisation and Reprocessing

Figure 1: PTSD Checklist mean total scores.

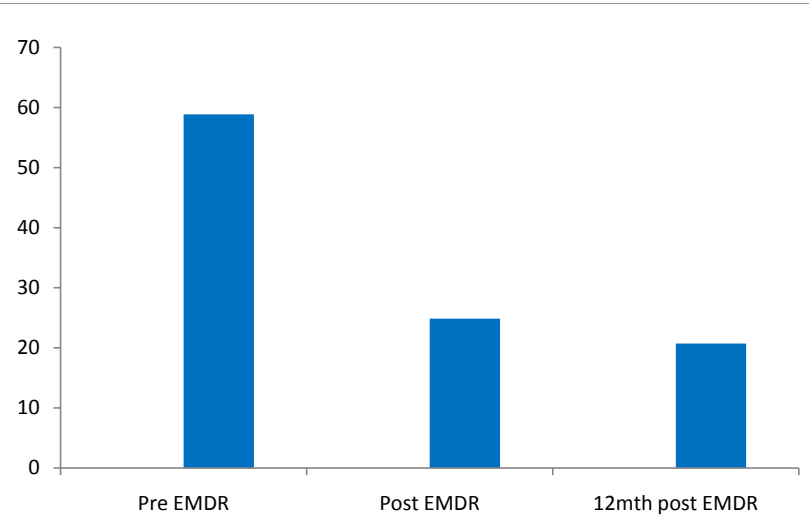

EMDR= Eye Movement Desensitisation and Reprocessing

Figure 2: Dissociative Experience Scale Percentage Mean Scores.

report measure (consisting of 28 items) to assess the degree to which people experience dissociation. VanIjzendroon and Schuengel [18] suggests the DES demonstrates impressive predictive validity, in particular, concerning dissociative disorder and traumatic experiences. In addition, Carlson et al. [19] found the DES a reliable and valid instrument to measure dissociation in many groups, and to have good concurrent validity for detecting severe dissociative disorders.

The SCID 2 is a semi-structured diagnostic interview used to make Axis II (personality disorders) diagnoses [11]. It is believed to be a time efficient questionnaire as the completion of the self-report screening questionnaire reduces the number of items needing evaluation by the clinician $[11,20]$. It can be used in both the research and clinical setting and has proven to be a reliable assessment tool [21].

The inclusion criteria for admission to and treatment at our clinic are a diagnosis of PTSD via clinical assessment and a score of $>44$ on the PTSD checklist (civilian version). Exclusion criteria are a primary diagnosis of substance dependence, significant organic brain disease, psychotic disorder or active suicidality.

All results were analysed using SPSS Statistics 20. Small sample sizes and categorical scores, except the DES scores, led to the use of non-parametric analyses for most tests.

\section{Results}

Of 31 patients referred 13 did not meet criteria for treatment at the clinic. Of the 18 patients accepted for treatment, 2 failed to complete treatment, 4 are ongoing in treatment, and 12 completed treatment with EMDR. Of the 12 who completed treatment, 12 month follow up data has been collected on 7 to date $(3$ males, 4 females mean age $=36 \mathrm{yrs}$, age range $=21-50$ ). Treatment was primarily for trauma occurring in adulthood in 6 patients ( 2 home invasions, one diving accident, one military - related, one physical assault, and one helicopter crash) and 3 of these also had histories of childhood traumas. The other patient had witnessed repeated severe domestic violence through her childhood and adolescence.

Mean number of EMDR sessions required was 4.71 (range 1-10)

The Repeated Measures One-Way ANOVA with a GreenhouseGeisser correction was used to assess within-subject differences between means. The focus here is on whether each participant improved their scores through treatment. All patients improved significantly on PTSD checklist scores (Repeat Measures One- Way ANOVA: $p<0.01$ ) and all were sub threshold for PTSD post treatment and at 12 month follow up (Figure 1).

Criterion symptom groups for the three PTSD symptom domains all showed improvement which was sustained at follow up.

Mean score for the DES improved significantly after EMDR (Figure 2 ) and continued to show significance in improvement at 12 month follow up(paired sample $\mathrm{t}$-test: $\mathrm{t}=4.292, \mathrm{p}<0.01$ ).

Wilcoxon Signed Ranks Tests were used to assess the mean difference between the two times of assessment (Pre-EMDR and 12 Month post EMDR) for the Hamilton Depression scale, the Hamilton Anxiety scale, and the SCID 2 data.

Improvements were noted on the Hamilton Depression scale for all 5 patients for whom this data was collected (Wilcoxon Signed Rank Test: $\mathrm{Z}=-2.032, \mathrm{P}=0.042$ ) and also for the Hamilton Anxiety scale $(\mathrm{Z}=11.384, \mathrm{P}=0.0005)$ and these improvements were also sustained at 12 month follow up (Figure 3 and 4).

The SCID 2 personality questionnaire scores are shown in Figure 5 and 6. Significant reductions sustained at 12 month follow-up were found for paranoid $(\mathrm{Z}=-2.060, \mathrm{P}=0.039)$, depressive $(\mathrm{Z}=-2.220$, $\mathrm{P}=0.026)$, and borderline traits $(\mathrm{Z}=-2.032, \mathrm{P}=0.042)$. In regard to personality disorder diagnoses via the SCID 2, 4 out of 7 patients had a diagnosis pre-treatment whereas only one still met criteria post treatment with EMDR and this patient no longer met criteria at 12 month follow up (Table 1).

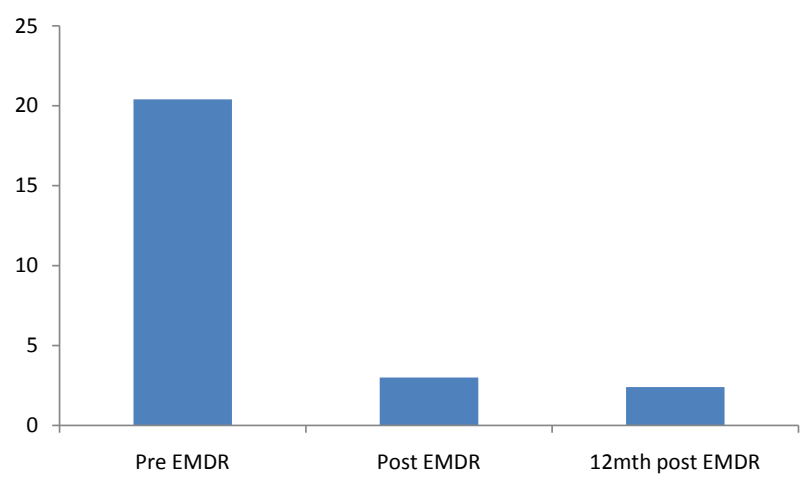

EMDR= Eye Movement Desensitisation and Reprocessing Figure 3: Hamilton Anxiety Scale Mean Scores. 
Citation: Laugharne J, Kullack C, Stanley S (2013) Eye Movement Desensitisation and Reprocessing Treatment of Posttraumatic Stress Disorder, Comorbid Disorders and Personality Traits: A Case Series with 12 Month Follow Up. J Trauma Treat S4: 003. doi:10.4172/2167-1222.S4003

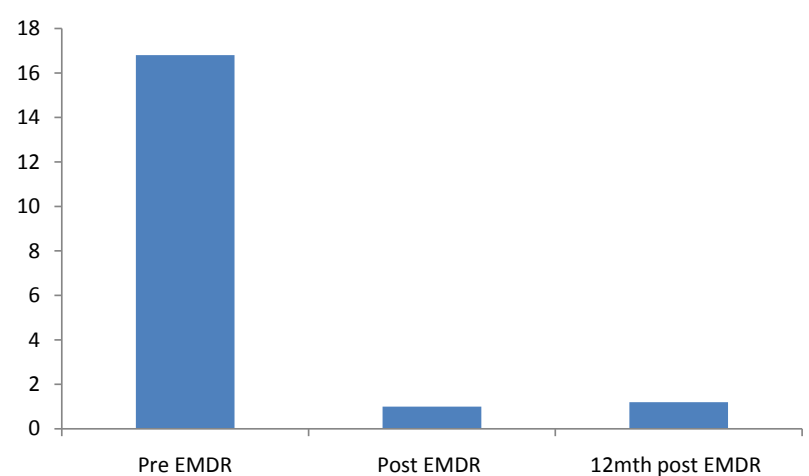

EMDR= Eye Movement Desensitisation and Reprocessing Figure 4: Hamilton Depression Scale Mean Scores.

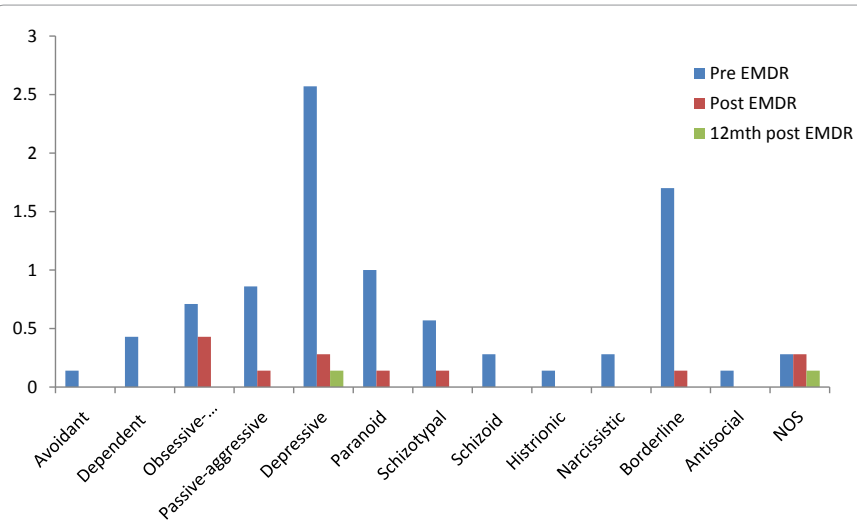

EMDR = Eye Movement Desensitisation and Reprocessing Figure 5: SCID 2 Personality Questionnaire Mean Scores.

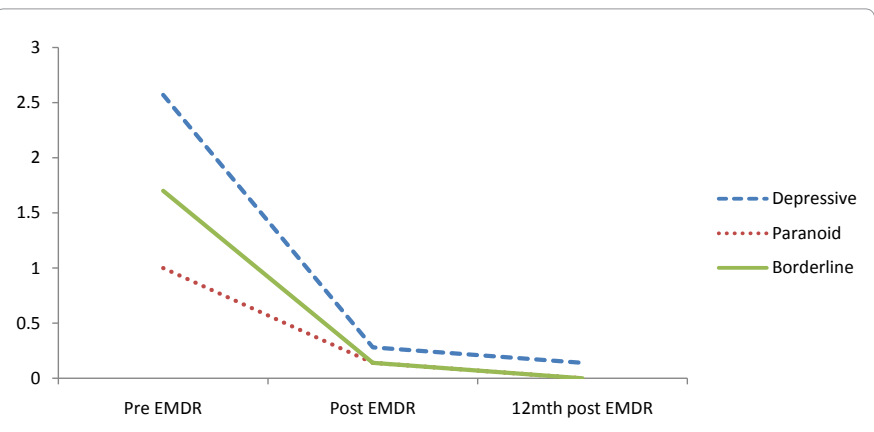

EMDR= Eye Movement Desensitisation and Reprocessing

Figure 6: SCID 2 Personality Questionnaire: Changes in Depressive, Paranoid and Borderline Mean Scores.

In regard to major comorbidities, data is available up to 12 month follow up for 5 of the patients. Results are presented in Table 2.

Finally, regarding concurrent psychopharmacological treatment during the relevant period, the medication prescribed to each patient is shown in Table 3.

\section{Discussion}

EMDR is internationally recognised as a gold standard treatment for PTSD [1-3]. EMDR uses bilateral stimulation to release process and desensitise traumatic material. The client maintains dual attention whilst connecting with the cognitive, somatic and emotional components of the trauma memory. The processing results in a reduction or cessation in the typical re-experiencing, avoidance and hyper-arousal PTSD symptoms enabling the trauma memory to move from a vivid memory to being recalled as a past or historical event [22].

Whilst the mechanism of action is unclear Stickgold [23] proposes that EMDR mimics the saccades of rapid eye movement sleep thus allowing unimpeded information processing to occur enabling the trauma memory to move from an episodic to semantic state. Similarly, Solomon and Shapiro [24] believe the bilateral stimulation and processing enables the trauma material to be assimilated with adaptive information in other memory networks. Hence, the trauma memory is no longer isolated but rather appropriately integrated.

A person requesting EMDR therapy must be referred to an accredited EMDR therapist who will complete a comprehensive assessment to ensure the person is suitable for EMDR therapy.

The findings reported here are of interest for a number of reasons. The improvement in PTSD symptoms following EMDR treatment has been well established through a series of randomised controlled trials [1] but the effects at 12 month follow up has not been so well documented. Our results suggest that patients treated with EMDR maintain their improvement 12 months post-treatment in terms of total symptom scores and across each of the criteria groupings. This latter finding suggests that EMDR may be effective in reducing not only re-experiencing symptoms but the range of symptoms experienced by these patients.

The rest of the findings taken together indicate that the treatment of PTSD with EMDR has measurable positive effects beyond the PTSD diagnosis. Significant improvements in Hamilton depression and anxiety scores were maintained at 12 month follow up. Whilst anxiety and depressive symptoms are relevant within the PTSD construct it is important to note that for the 5 patients administered the MINI plus at baseline, 3 had comorbid Major Depressive Episode, 2 had comorbid Panic Disorder and 2 met criteria for both Agoraphobia and Social Phobia. This is consistent with the well recognised fact that PTSD patients have high rates of comorbidity [4,5]. However, we found that post treatment only one out of the four patients with a comorbid anxiety or mood disorder still had such a comorbidity post treatment and this was still the case at 12 month follow up.

Dissociative symptoms are common in traumatised individuals $[25,26]$ and can have significant impact on functioning and indeed can interfere with the treatment of posttraumatic symptoms. We have found significant improvement in dissociative symptoms measured via

\begin{tabular}{|c|c|c|c|c|}
\hline PT & $\begin{array}{c}\text { PRE EMDR } \\
\text { THERAPY }\end{array}$ & $\begin{array}{c}\text { POST EMDR } \\
\text { THERAPY }\end{array}$ & $\begin{array}{c}\text { 6MTH POST EMDR } \\
\text { THERAPY }\end{array}$ & $\begin{array}{c}\text { 12MTH POST } \\
\text { EMDR THERAPY }\end{array}$ \\
\hline 1 & Depressive PD & Nil & Nil & Nil \\
\hline 2 & $\begin{array}{c}\text { PD Not Otherwise } \\
\text { specified }\end{array}$ & $\begin{array}{c}\text { PD Not } \\
\text { Otherwise } \\
\text { Specified }\end{array}$ & $\begin{array}{c}\text { PD Not Otherwise } \\
\text { Specified }\end{array}$ & Nil \\
\hline 3 & Nil & Nil & Nil & Nil \\
\hline 4 & Nil & Nil & Nil & Nil \\
\hline 5 & $\begin{array}{c}\text { Avoidant PD } \\
\text { Borderline PD }\end{array}$ & Nil & Nil & Nil \\
\hline 6 & Avoidant PD & Nil & Nil & Nil \\
\hline 7 & Nil & Nil & Nil & Nil \\
\hline
\end{tabular}

EMDR= Eye Movement Desensitisation and Reprocessing $\mathrm{PD}=$ Personality Disorder

Table 1: SCID 2 Personality Questionnaire Results. 
Citation: Laugharne J, Kullack C, Stanley S (2013) Eye Movement Desensitisation and Reprocessing Treatment of Posttraumatic Stress Disorder, Comorbid Disorders and Personality Traits: A Case Series with 12 Month Follow Up. J Trauma Treat S4: 003. doi:10.4172/2167-1222.S4003

Page 4 of 5

\begin{tabular}{|c|c|c|c|c|}
\hline PT & PRE EMDR THERAPY & POST EMDR THERAPY & 6MTH POST EMDR THERAPY & 12MTH POST EMDR THERAPY \\
\hline 1 & $\begin{array}{c}\text { PTSD } \\
\text { Substance dependence past } 12 \mathrm{mths}\end{array}$ & Substance dependence past $12 \mathrm{mths}$ & Substance dependence past $12 \mathrm{mths}$ & Nil \\
\hline 2 & $\begin{array}{l}\text { MDE with melancholic features } \\
\text { Suicidality -moderate risk } \\
\text { Panic disorder } \\
\text { PTSD } \\
\text { Alcohol dependence past } 12 \mathrm{mths}\end{array}$ & Nil & Nil & Nil \\
\hline 3 & $\begin{array}{l}\text { MDE } \\
\text { PTSD }\end{array}$ & Nil & Nil & Nil \\
\hline 4 & $\begin{array}{l}\text { MDE with melancholic features } \\
\text { Suicidality -low risk } \\
\text { Panic disorder } \\
\text { Agoraphobia } \\
\text { Social phobia } \\
\text { Specific phobia } \\
\text { PTSD }\end{array}$ & Nil & Nil & Nil \\
\hline 6 & $\begin{array}{c}\text { Panic disorder } \\
\text { Agoraphobia } \\
\text { Social phobia } \\
\text { PTSD } \\
\text { Alcohol dependence past } \\
\text { 12mths }\end{array}$ & $\begin{array}{c}\text { Panic disorder } \\
\text { Agoraphobia } \\
\text { Alcohol dependence past 12mths }\end{array}$ & $\begin{array}{c}\text { Panic disorder } \\
\text { Agoraphobia } \\
\text { Alcohol dependence past 12mths }\end{array}$ & $\begin{array}{c}\text { Panic disorder } \\
\text { Agoraphobia } \\
\text { Alcohol dependence past 12mths }\end{array}$ \\
\hline
\end{tabular}

EMDR= Eye Movement Desensitisation and Reprocessing

PTSD $=$ Posttraumatic Stress Disorder

MDE=Major Depressive Disorder

Table 2: MINI PLUS Results.

\begin{tabular}{|c|c|c|c|c|}
\hline PT & PRE EMDR THERAPY & POST EMDR THERAPY & 6MTH POST EMDR THERAPY & 12MTH POST EMDR THERAPY \\
\hline 1 & Sertraline $300 \mathrm{mgs}$ & Sertraline $300 \mathrm{mgs}$ & Sertraline 200 mgs \\
\hline 2 & Escitalopram $10 \mathrm{mgs}$ & Escitalopram $10 \mathrm{mgs}$ & Escitalopram $10 \mathrm{mgs}$ \\
\hline 3 & Nil antidepressant therapy & Nil antidepressant therapy & Nil antidepressant therapy & Nil antidepressant therapy \\
\hline 4 & Citalopram $40 \mathrm{mgs}$ & Citalopram $40 \mathrm{mgs}$ & Nil antidepressant therapy & Nil antidepressant therapy \\
\hline 5 & Mirtazepine $15 \mathrm{mgs}$ & Mirtazepine $15 \mathrm{mgs}$ & Nil antidepressant therapy \\
\hline 6 & Venlafexine150 mgs & Venlafexine & Venlafexine \\
\hline 7 & Nil antidepressant therapy & Nil antidepressant therapy & Nil antidepressant therapy & Venlafexine \\
\hline
\end{tabular}

EMDR $=$ Eye Movement Desensitisation and Reprocessing

Table 3: Patients'Antidepressant Therapy.

the DES, maintained at 12 month follow up. This is, to our knowledge, the first time that such a finding has been reported.

The results from our SCID 2 evaluations are particularly interesting. Personality Disorders are by definition considered to be longstanding, ingrained and therefore resistant to change although in recent years there has been evidence for some treatment optimism [27]. Furthermore there is evidence for associations between trauma history and certain personality disorders notably Borderline Personality Disorder [28] and Paranoid Personality Disorder [29]. There is therefore a rationale for expecting that effective treatment of PTSD may have the added effect of reducing pathological personality traits which are traumatic in origin. There is very little in the existing literature which reports on this possibility. Brown and Shapiro [30] have reported on a single case of Borderline Personality Disorder who showed notable improvement in pathological personality traits with EMDR treatment of past traumas. Our findings, whilst preliminary, support such a rationale both in terms of sustained trait reductions and personality disorder diagnoses. Our findings, that four out of seven patients had a Personality Disorder diagnosis via SCID 2 prior to treatment, whereas no patient retained such a diagnosis 12 months post treatment are encouraging from a therapeutic perspective.

Limitations of these data include the relatively small number of patients, the lack of a control group and the administration of instruments by one of the treating clinicians. Furthermore, data gathered from a case-series is less readily generalised than data from studies with more systematic methodologies. One advantage of such a series, however, is that the patients are "real life" PTSD Clinic patients, not highly selected, as is often the case in research cohorts.

In conclusion, although the number of patients described in this paper is small, our data suggest that EMDR may be effective in reducing the symptoms of psychiatric disorders comorbid with PTSD including Personality Disorders. Current treatment approaches for the latter, such as Dialectical Behaviour Therapy for Borderline Personality Disorder is relatively resource intensive. Our findings suggest that EMDR may be a treatment modality which could reduce the need for such prolonged therapy by simultaneously treating PTSD and the symptoms of Borderline Personality Disorder thus contributing to more effective clinical management. That our findings are significant in spite of limited numbers suggests this to be potentially fertile ground for further research with appropriate controls.

\section{References}

1. Bisson JI, Ehlers A, Matthews R, Pilling S, Richards D, et al. (2007) Psychological treatments for chronic post-traumatic stress disorder. Systematic review and meta-analysis. Br J Psychiatry 190: 97-104.

2. American Psychiatric Association (2004) Practical guidelines for the treatment of patients with acute stress disorder and posttraumatic stress disorder Arlington VA: American Psychiatric Association Practice Guidelines.

3. Forbes D, Creamer M, Phelps A, Bryant R, McFarlane A, et al. (2007) Australian 
Citation: Laugharne J, Kullack C, Stanley S (2013) Eye Movement Desensitisation and Reprocessing Treatment of Posttraumatic Stress Disorder, Comorbid Disorders and Personality Traits: A Case Series with 12 Month Follow Up. J Trauma Treat S4: 003. doi:10.4172/2167-1222.S4003

guidelines for the treatment of adults with acute stress disorder and posttraumatic stress disorder. Aust N Z J Psychiatry 41: 637-648.

4. Grant DM, Beck G, Marques L, Palyo SA, Clapp JD (2008) The structure of distress following trauma: posttraumatic stress disorder, major depressive disorder and generalized anxiety disorder. J Abnorm Psychol 117: 662-672.

5. Pietrzak RH, Goldstein RB, Southwick SM, Grant BF (2011) Prevalence and Axis I comorbidity of full and partial posttraumatic stress disorder in the United States: results from Wave 2 of the National Epidemiologic Survey on Alcohol and Related Conditions. J Anxiety Disord 25: 456-465.

6. Sheehan DV, Lecrubier Y, Sheehan KH, Amorim P, Janavs J, et al. (1998) The Mini-International Neuropsychiatric Interview (M.I.N.I.): the development and validation of a structured diagnostic psychiatric interview for DSM-IV and ICD10. J Clin Psychiatry 59 Suppl 20: 22-33.

7. Blanchard EB, Jones-Alexander J, Buckley TC, Forneris CA (1996) Psychometric properties of the PTSD Checklist (PCL). Behav Res Ther 34: 669-673.

8. Hamilton M (1959) The assessment of anxiety states by rating. $\mathrm{Br} \mathrm{J} \mathrm{Med}$ Psychol 32: 50-55

9. Hamilton M (1960) A rating scale for depression. J Neurol Neurosurg Psychiatry 23: $56-62$.

10. Bernstein EM, Putnam FW (1986) Development, reliability, and validity of a dissociation scale. J Nerv Ment Dis 174: 727-735.

11. First MB, Gibbon M, Spitzer RL, Williams JBW \& Benjamin LS (1997) User's guide for the Structured Clinical Interview for DSM IVAxis II Personality Disorders (SCID II). Washington DC, American Psychiatric Press.

12. Sheehan DV, Lecrubier Y, Harnett-Sheehan K (1997) The validity of the MiniInternational Neuropsychiatric Interview(MINI)according to the SCID-P and it's reliability. European Psychiatry 12: 232-241.

13. Lecrubier Y, Sheehan DV, Weiller E (1997) The Mini Internationa Neuropsychiatric Interview (MINI). A short diagnosticstructured interview: reliability and validity according to the CIDI. European Psychiatry 12: 224-231.

14. McDonald SD, Calhoun PS (2010) The diagnostic accuracy of the PTSD checklist: a critical review. Clin Psychol Rev 30: 976-987.

15. Maier W, Buller R, Philipp M, Heuser I (1988) The Hamilton Anxiety Scale: reliability, validity and sensitivity to change in anxiety and depressive disorders. J Affect Disord 14: 61-68.

16. Bagby RM, Ryder AG, Schuller DR, Marshall MB (2004) The Hamilton Depression Rating Scale: has the gold standard become a lead weight? Am J Psychiatry 161: 2163-2177.
17. Bech P, Allerup P, Gram LF, Reisby N, Rosenberg R, et al. (1981) The Hamilton depression scale. Evaluation of objectivity using logistic models. Acta Psychiatr Scand 63: 290-299.

18. Van ljzendoorn $\mathrm{MH}$, Schuengel C (1996) The measurement of dissociation in normal and clinical populations: meta-analyticvalidation of the Dissociative Experience Scale (DES). Clinical Psychology Review 16: 365-382.

19. Carlson EB, Putnam FW, Ross CA, Torem M, Coons P, et al. (1993) Validity of the Dissociative Experiences Scale in screening for multiple personality disorder: a multicenter study. Am J Psychiatry 150: 1030-1036.

20. Ekselius L, Lindström E, von Knorring L, Bodlund O, Kullgren G (1994) SCID II interviews and the SCID Screen questionnaire as diagnostic tools for personality disorders in DSM-III-R. Acta Psychiatr Scand 90: 120-123.

21. Jacobsberg L, Perry S, Frances A (1995) Diagnostic agreement between the SCID-II screening questionnaire and the Personality Disorder Examination. J Pers Assess 65: 428-433.

22. Shapiro, F (2001) Eye movement desensitisation and reprocessing: Basic principles, protocols, and procedures (2ndedn), New York: Guildford Press.

23. Stickgold R (2002) EMDR: a putative neurobiological mechanism of action. $J$ Clin Psychol 58: 61-75

24. Solomon RM, Shapiro F (2008) EMDR and the adaptive information processing model: potential mechanisms of change. Journal of EMDR Practice and Research 1: 109-117.

25. Haddock DB (2001) The dissociative identity disorder sourcebook. New York: McGraw-Hill.

26. Murray J, Ehlers A, Mayou RA (2002) Dissociation and post-traumatic stress disorder: two prospective studies of road traffic accident survivors. $\mathrm{Br}$ Psychiatry 180: 363-368.

27. Stone MH (2006) Management of borderline personality disorder: a review of psychotherapeutic approaches. World Psychiatry 5: 15-20.

28. Lee R (2006) Childhood trauma and personality disorder: toward a biological model. Curr Psychiatry Rep 8: 43-52.

29. Rademaker AR, Vermetten E, Geuze E, Muilwijk A, Kleber RJ (2008) Selfreported early trauma as a predictor of adult personality: a study in a military sample. J Clin Psychol 64: 863-875.

30. Brown S, Shapiro F (2006) EMDR in the treatment of borderline personality disorder. Clinical Case Studies 5: 403-420.
This article was originally published in a special issue, Post Traumatic Stress Disorders handled by Editor(s). Dr. Agius M, University of Cambridge, UK 